$16^{\text {th }}$ International Congress of Metrology, 03001 (2013)

DOI: $10.1051 /$ metrology/201303001

(c) Owned by the authors, published by EDP Sciences, 2013

\title{
CARACTÉRISATION MÉTROLOGIQUE D'UNE NOUVELLE RÉFÉRENCE DE PRESSION RELATIVE DANS LE DOMAINE DE 1,5 À 50 MPA
}

\author{
Thierry Rabault $^{1 \mathrm{a}}$, Padipat Wongthep ${ }^{12 \mathrm{~b}}$ et Djilali Bentouati ${ }^{1 \mathrm{c}}$ \\ ${ }^{1}$ Laboratoire National de Métrologie et d'Essais, 1 rue Gaston Boissier - F 75724 Paris Cedex 15, France \\ ${ }^{2}$ National Institute of Metrology (Thailand), 3/4-5 Moo 3 Tambol Klonghar Amphur Klong Luang, Thailand
}

\begin{abstract}
Résumé. La nouvelle référence de pression relative dans le domaine de 1,5 à $50 \mathrm{MPa}$ est définie à partir de trois ensembles piston-cylindres (EPC). Ces ensembles de $50 \mathrm{kPa} / \mathrm{kg}(15,8 \mathrm{~mm}$ de diamètre) peuvent être utilisés en jeu contrôlé et sont adaptés pour être montés sur les balances de référence haute pression d'huile, équipées chacune d'un ensemble de masses de $1000 \mathrm{~kg}$.

La section effective du premier EPC a été déterminée par des mesures dimensionnelles avec une incertitude relative élargie $(k=2)$ estimée à $1,0 \times 10^{-5}$, puis comparée aux autres ensembles. Par ailleurs, les résultats dimensionnels ont été confirmés à basse pression par comparaison directe avec la référence de $1 \mathrm{MPa}$.

Les coefficients de déformation des ensembles piston-cylindre ont été déterminés expérimentalement avec un ensemble $200 \mathrm{MPa}$, et comparés aux valeurs théoriques calculées par la méthode des éléments finis (MEF).

Le bilan d'incertitude sur la mesure de pression pour cette référence est présentée. L'incertitude élargie est estimée à $15 \mathrm{~Pa}+1,2 \times 10^{-5} \mathrm{x}$.
\end{abstract}

\begin{abstract}
The new LNE gauge pressure standard in the range 1,5 to $50 \mathrm{MPa}$, is composed of three pistoncylinders assemblies (PCA). These are $50 \mathrm{kPa} / \mathrm{kg}$ controlled clearance piston-cylinder (diameter of 15,8 $\mathrm{mm}$ ) designed to be adapted on the oil-operated high-pressure reference balances using a $1000 \mathrm{~kg}$ mass set.

The effective area of the first PCA was determined by dimensional measurements with a expanded $(k=2)$ relative uncertainty estimated to be $1.0 \times 10^{-5}$, and then compared with the other ones. Moreover, the dimensional results were confirmed in the lower pressure range by direct comparison with the $1 \mathrm{MPa}$ reference. The distortion coefficients of the piston-cylinder assemblies were experimentally determined with the $200 \mathrm{MPa}$ unit, and compared with the values calculated by Finite Element Method (FEM).

The pressure measurement uncertainty budget for this standard is detailed. The expanded uncertainty is estimated to be $15 \mathrm{~Pa}+1.2 \times 10^{-5} \mathrm{x}$.
\end{abstract}

\section{Contexte et objectifs}

La chaîne de référence haute pression du Laboratoire National de métrologie et d'Essais (LNE) a vu le jour en 1976 avec la réalisation de deux balances de pression comportant chacune un jeu de masses de $1000 \mathrm{~kg}$, sur lesquelles ont été adaptés des blocs de mesures permettant de recevoir des ensembles piston-cylindre de $50 \mathrm{~mm}^{2}$.pour atteindre une pression de $200 \mathrm{MPa}$ [1]. En 1984, cette chaîne a été étendue jusqu'à 1 GPa avec des ensembles piston-cylindre de $10 \mathrm{~mm}^{2}$.

Cependant, la masse minimale applicable sur ces balances est de $30 \mathrm{~kg}$, ce qui implique un premier palier de pression à $6 \mathrm{MPa}$ et la pression maximale mesurée par nos références primaires ne dépasse pas $1 \mathrm{MPa}$. nous n'avons par conséquent pas de recouvrement entre nos étalons primaires et notre chaîne de référence haute pression. C'est pourquoi, nous avons décidé de développer des étalons de $200 \mathrm{~mm}^{2}$ qui nous permettra d'atteindre une pression maximale de $50 \mathrm{MPa}$, et de démarrer avec une pression de 1,5 $\mathrm{MPa}$.

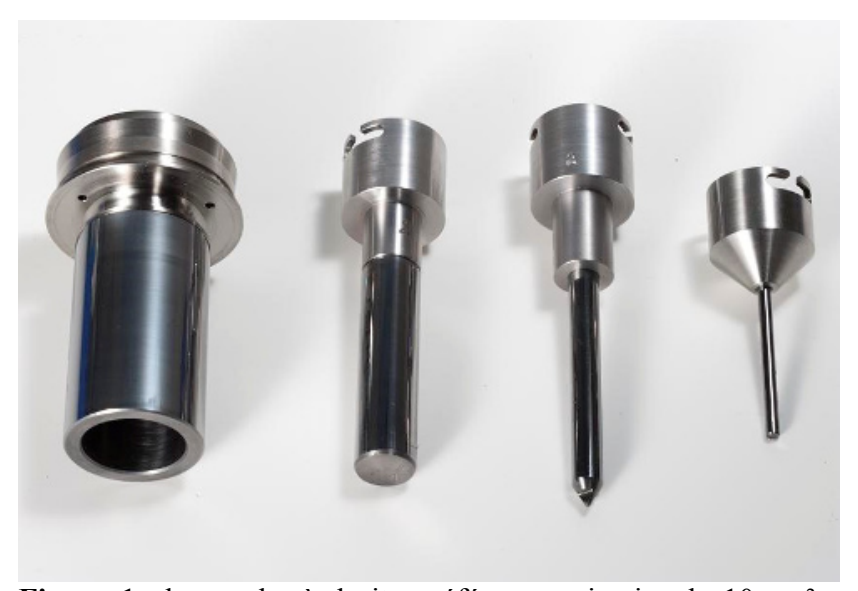

Figure 1. de gauche à droite : référence primaire de $10 \mathrm{~cm}^{2}$ (1 MPa), piston 50, 200 et $1000 \mathrm{MPa}$.

\footnotetext{
a thierry.rabault@lne.fr

b patipat@nimt.or.th

c djilali.bentouati@lne.fr
}

This is an Open Access article distributed under the terms of the Creative Commons Attribution License 2.0, which permits unrestricted use, distribution, and reproduction in any medium, provided the original work is properly cited. 
Du fait de leurs grands diamètres $(15,8 \mathrm{~mm})$, ces ensembles permettront d'obtenir à partir de mesures dimensionnelles une incertitude-type de 5 ppm sur la détermination de la section effective, avec une incertitude élargie de $80 \mathrm{~nm}$ sur les mesures de diamètre. Ces ensembles deviendront ainsi nos références primaires pour le domaine des hautes pressions.

\section{Définition et unités}

La pression $P$ est définie comme une force appliquée à une surface. La surface est matérialisée par des ensembles piston-cylindre de section $S_{\mathrm{p}}$ et la force par une masse $M$ déposée sur le piston soumise à l'accélération de la pesanteur $g$. Son unité dans le système international $(\mathrm{SI})$ est le pascal $(\mathrm{Pa})$ :

$$
P=\frac{M g}{S_{p}}
$$

Sous l'action de la pression, le piston et le cylindre ont tendance à se déformer, entrainant ainsi une variation de la section qui généralement est linéaire et peut s'écrire sous la forme :

$$
S_{p}=S_{0}(1+\lambda P)
$$

$S_{0}$ est la section effective à pression nulle ramenée à une température de $20^{\circ} \mathrm{C}$ et $\lambda$ le coefficient de déformation exprimé en $\mathrm{Pa}^{-1}$.

\section{Théorie des balances de pression}

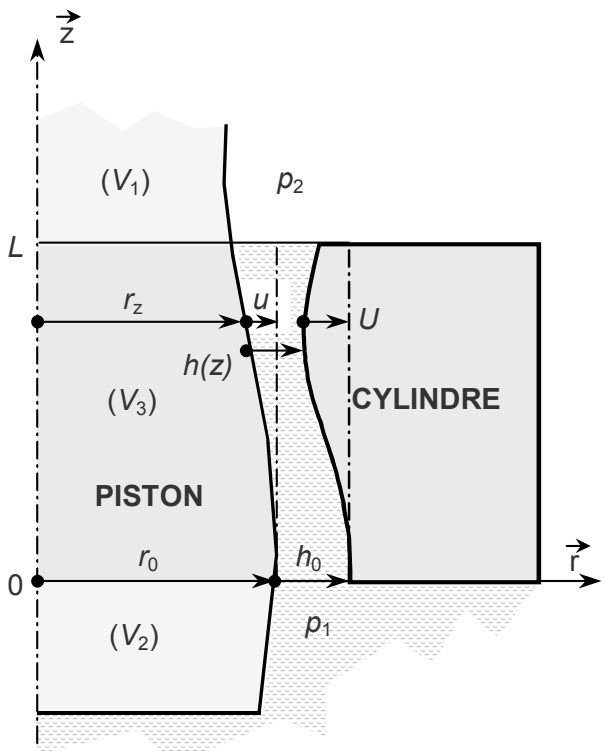

Figure 2. Diagramme en coupe d'un ensemble piston-cylindre et notations.

Le modèle associé aux balances de pression [2], est calculé à partir de l'équilibre des forces appliquées au piston.

$$
F_{g}+F_{z}+F_{V}=0
$$

où $F_{\mathrm{g}}$ est la force de pesanteur, $F_{\mathrm{z}}$ la force hydrostatique et $F_{\mathrm{v}}$ la force de viscosité.

La force de pesanteur est égale à la somme du produit des masses déposées mi par l'accélération de la pesanteur $g$, qui est de $9,809273 \mathrm{~m} / \mathrm{s}^{2}$ au niveau du laboratoire de métrologie des pressions du LNE :

$$
F_{g}=-\sum_{i} m_{i} g .
$$

La force hydrostatique est la résultante des forces appliquées sur le piston lorsqu'il est plongé dans un liquide. Pour la calculer, nous devons découper le piston en trois sous domaines (Figure 2) de volumes respectifs : $\left(V_{1}\right)$ la partie émergente du piston qui est soumise à la pression $p_{2}$,

$\left(V_{2}\right)$ la partie inférieure du piston qui est située dans la chambre et qui est maintenue à la pression de mesure $p_{1}$, $\left(V_{3}\right)$ la zone d'engagement du piston dans le cylindre dans laquelle la pression varie entre la pression de mesure $p_{1}$ et la pression $p_{2}$.

$F_{\mathrm{z}}$ est donnée par l'équation (5), ci dessous dans laquelle $u$ est la déformée du piston et $\rho\left(p_{1}\right)$ et $\rho_{\mathrm{a}}$ sont les masses volumiques du fluide respectivement dans la chambre et à la pression atmosphérique :

$$
\begin{array}{r}
F_{z}=\rho\left(p_{1}\right) g V_{1}+\rho_{a} g V_{2}-\pi r_{0}^{2}\left(p_{2}-p_{1}\right)+\cdots \\
2 \pi r_{0} \int_{0}^{L}\left(p(z)-p_{2}\right) \frac{d u}{d z} d z
\end{array} .
$$

Pour calculer la force de viscosité, nous utilisons l'équation de Navier-Stokes dans laquelle nous considèrerons que la vitesses $v$ de l'écoulement est stationnaire $\partial v / \partial t=0$ et absent de forces de masses.

Dans le cas où le fluide est incompressible $\operatorname{div} \vec{v}=0$ et que les termes d'inertie sont négligeables, nous obtenons l'équation de Reynolds (6) qui s'applique aux fluides visqueux s'écoulant lentement dans un passage étroit tel que le jeu compris entre le piston et le cylindre :

$$
\eta \Delta \vec{v}-\overrightarrow{\operatorname{grad}} p=0
$$

$\eta$ le coefficient de viscosité dynamique exprimé en Pa.s.

Pour résoudre cette équation, nous assimilerons le jeu à deux parois parallèles. Nous pouvons ainsi en déduire la vitesse d'écoulement du fluide dans le jeu qui nous permet ensuite de calculer la force de viscosité $F_{\mathrm{v}}$ et le débit $Q$ s'écoulant à travers le jeu entre piston et cylindre. 


$$
\begin{gathered}
F_{v}=-\pi r_{0} \int_{0}^{L} \frac{d p}{d z} h(z) d z, \\
Q=-\pi r \frac{h^{3}}{6 v} \frac{d p}{d z},
\end{gathered}
$$

où $v=\eta / \rho$ est la viscosité cinématique du fluide.

Nous définissons la hauteur de référence $h_{\text {réf }}$ du piston comme le rapport du volume du piston situé dans la chambre de pression $V_{1}$ par la section du cylindre $S_{\mathrm{p}}(9)$ et le volume $V_{2}$ situé sur la partie supérieure du cylindre est égal la somme des masses divisées par leurs masses volumiques respectives :

$$
\begin{gathered}
h_{\text {réf }}=-\frac{V_{1}}{S_{p}}, \\
V_{2}=\sum_{i} \frac{m_{i}}{\rho_{m_{i}}} .
\end{gathered}
$$

Dans l'équation d'équilibre (3), nous introduisons les expressions des forces (4), (5) et (7) trouvées précédemment et nous obtenons ainsi l'expression de la pression qui en pression relative $\left(p_{2}=P_{\mathrm{atm}}, p_{1}=P P_{\mathrm{atm}}\right)$ prend la forme suivante :

$$
P=\frac{\sum_{i} m_{i}\left(1-\frac{\rho_{a}}{\rho_{m_{i}}}\right) g}{S_{p}}+\rho\left(P-P_{\text {atm }}\right) g h_{\text {réf }} .
$$

Après avoir écrit que $h(z)=h_{0}+U-u$ nous obtenons la section effective $S_{\mathrm{p}}$ :

$$
\begin{aligned}
& S_{p}=\pi r_{0}^{2}\left[1+\frac{h_{0}}{r_{0}}+\cdots\right. \\
& \left.\frac{1}{r_{0}\left(p_{1}-p_{2}\right)} \int_{0}^{L}\left(p(z)-p_{2}\right) \frac{d(U+u)}{d z} d\right]
\end{aligned}
$$

Le champ de pression $p_{\mathrm{z}}$ (13) est calculé en considérant que le débit reste constant le long du jeu :

$$
p_{z}=P\left[1-\int_{0}^{z} \frac{v}{h^{3}} d z / \int_{0}^{L} \frac{v}{h^{3}} d z\right]
$$

La section effective à pression nulle peut alors être calculée en considérant que $S_{0}$ est la limite de $S_{\mathrm{p}}$ lorsque $p_{1}$ tend vers $p_{2}$. Dans ces conditions, la viscosité est constante sur toute la longueur du jeu, ce qui nous permet d'écrire :

$$
S_{0}=\pi r_{0}^{2}\left[1+\frac{h_{0}}{r_{0}}+\frac{1}{r_{0}} \int_{0}^{L} \frac{(u+U)}{h^{3}} d z / \int_{0}^{L} \frac{1}{h^{3}} d z\right] .
$$

Lorsque l'on néglige les défauts de formes du piston et du cylindre $u$ et $U$, alors nous pouvons considérer que les rayons $r_{0}$ et $R_{0}$ sont respectivement égaux aux rayons moyens $\bar{r}$ et $\bar{R}$, ce qui nous donne une expression simplifiée de la section effective à pression nulle :

$$
S_{0}=\pi \bar{R} \bar{r} .
$$

\section{Mesures dimensionnelles}

Les mesures dimensionnelles ont initialement été réalisées en 2007 sur l'ensemble $50 \mathrm{MPa} \mathrm{N}^{\circ} 1$. Elles ont été effectuées suivant deux génératrices $0-180^{\circ}$ et $90-270^{\circ}$ et pour trois altitudes 7,23 et $39 \mathrm{~mm}$. Chaque combinaison génératrice/altitude a été répétée entre 6 et 10 fois et refaite sur plusieurs jours. Ce qui au final, nous donne un nombre d'échantillons pouvant varier entre 18 et 69. Le tableau 1 nous donne la valeur moyenne, l'écart-type et le nombre d'échantillons pour chaque génératrice et altitude du piston et du cylindre. La valeur des rayons piston et cylindre est égale à la moyenne pondérée des demi-diamètres. On trouve ainsi pour le piston un rayon de $7,900623 \mathrm{~mm}$ avec une reproductibilité de $0,000016 \mathrm{~mm}$ et pour le cylindre un rayon de $7,900707 \mathrm{~mm}$ avec une reproductibilité de $0,000017 \mathrm{~mm}$.

Les diamètres extérieurs du piston ont été mesurés par comparaison interférométriques par rapport à un jeu de cales étalon acier de $15 \mathrm{~mm}$ pour et pour le diamètre intérieur du cylindre par rapport à un pont de cales étalon acier de $10,5 \mathrm{~mm}$. L'incertitude sur la mesure de diamètre obtenue par cette méthode est de $80 \mathrm{~nm}(2 \sigma)$.

Ces mesures ont été réalisées à nouveau en 2011, sur cinq altitudes différentes: 2, 11, 20, 29 et $38 \mathrm{~mm}$ (Tableau 2). On trouve alors pour le piston un rayon de $7,900598 \mathrm{~mm}$ avec une reproductibilité de 0,000 $14 \mathrm{~mm}$ et un rayon de $7,900715 \mathrm{~mm}$ avec une reproductibilité de $0,00015 \mathrm{~mm}$. Ce qui nous donne un écart par rapport aux mesures de 2007 de $25 \mathrm{~nm}$ sur le piston et $9 \mathrm{~nm}$ sur le cylindre.

À partir de la relation (15) nous pouvons déterminer la section et son incertitude associée $(1 \sigma)$, ainsi que la valeur du jeu moyen (Tableau 3).

Tableau 3. Valeurs de la section effective à pression nulle et jeu moyen de l'ensemble piston-cylindre $50 \mathrm{MPa} \mathrm{N}^{\circ} 1$.

\begin{tabular}{ccc}
\hline \hline & So $/ \mathrm{mm}^{2}$ & ho $/ \mu \mathrm{m}$ \\
\hline \hline 2007 & $196,09981 \pm 0,00091$ & $0,083 \pm 0,037$ \\
2011 & $196,09941 \pm 0,00087$ & $0,117 \pm 0,035$ \\
\hline \hline moyenne : & $196,09961 \pm 0,00013$ & $0,100 \pm 0,050$ \\
\cline { 2 - 3 }
\end{tabular}

Le profil des rayons piston et cylindre (Figure 3) ont été calculés à partir de la moyenne des rectitudes mesurées selon les génératrices $0,90,180$ et $270^{\circ}$, que nous avons ajustées par rapport aux demi-diamètres issus du tableau 2. 
Tableau 1. Mesures de diamètre réalisées en 2007 sur le piston et cylindre $50 \mathrm{MPa} \mathrm{N}{ }^{\circ} 1$.

\begin{tabular}{cccccccc} 
& \multicolumn{3}{c}{ Cylindre } & & \multicolumn{2}{c}{ Piston } \\
\hline \hline Génératrice & $\mathrm{z}$ & $\varnothing$ moyen & $\begin{array}{c}\text { Ecart-type } \\
\mathrm{mm}\end{array}$ & $\begin{array}{c}\text { Nombre } \\
\text { échantillons }\end{array}$ & $\begin{array}{c}\varnothing \text { moyen } \\
\mathrm{mm}\end{array}$ & $\begin{array}{c}\text { Ecart-type } \\
\text { mm }\end{array}$ & $\begin{array}{c}\text { Nombre } \\
\text { échantillons }\end{array}$ \\
\hline \hline $0-180^{\circ}$ & 10 & 15,801556 & 0,000033 & 29 & 15,801350 & 0,000047 & 49 \\
& 20 & 15,801487 & 0,000029 & 20 & 15,801196 & 0,000023 & 18 \\
& 29 & 15,801288 & 0,000031 & 51 & 15,801207 & 0,000029 & 25 \\
\hline $90-270^{\circ}$ & 10 & 15,801566 & 0,000033 & 26 & 15,801346 & 0,000039 & 69 \\
& 20 & 15,801509 & 0,000027 & 23 & 15,801186 & 0,000022 & 18 \\
& 29 & 15,801298 & 0,000031 & 47 & 15,801192 & 0,000028 & 20 \\
\hline \hline
\end{tabular}

Tableau 2. Mesures de diamètre réalisées en 2011 sur le piston et cylindre $50 \mathrm{MPa} \mathrm{N}^{\circ} 1$.

\begin{tabular}{|c|c|c|c|c|c|c|c|}
\hline \multirow[b]{2}{*}{ Génératrice } & \multirow[b]{2}{*}{$\begin{array}{c}\mathrm{Z} \\
\mathrm{mm}\end{array}$} & \multicolumn{3}{|c|}{ Cylindre } & \multicolumn{3}{|c|}{ Piston } \\
\hline & & $\begin{array}{c}\text { moyen } \\
\text { mm }\end{array}$ & $\begin{array}{c}\text { Ecart-type } \\
\mathrm{mm}\end{array}$ & $\begin{array}{c}\text { Nombre } \\
\text { échantillons }\end{array}$ & $\begin{array}{c}\varnothing \text { moyen } \\
\mathrm{mm}\end{array}$ & $\begin{array}{c}\text { Ecart-type } \\
\mathrm{mm}\end{array}$ & $\begin{array}{c}\text { Nombre } \\
\text { échantillons }\end{array}$ \\
\hline \multirow[t]{5}{*}{$0-180^{\circ}$} & 2 & 15,801522 & 0,000034 & 15 & 15,801249 & 0,000027 & 14 \\
\hline & 11 & 15,801523 & 0,000027 & 14 & 15,801237 & 0,000033 & 16 \\
\hline & 20 & 15,801468 & 0,000026 & 14 & 15,801144 & 0,000027 & 15 \\
\hline & 29 & 15,801340 & 0,000029 & 14 & 15,801176 & 0,000023 & 15 \\
\hline & 38 & 15,801270 & 0,000026 & 13 & 15,801148 & 0,000027 & 13 \\
\hline \multirow[t]{5}{*}{$90-270^{\circ}$} & 2 & 15,801536 & 0,000030 & 14 & 15,801219 & 0,000017 & 13 \\
\hline & 11 & 15,801520 & 0,000030 & 11 & 15,801256 & 0,000034 & 13 \\
\hline & 20 & 15,801517 & 0,000033 & 12 & 15,801185 & 0,000025 & 13 \\
\hline & 29 & 15,801355 & 0,000022 & 12 & 15,801187 & 0,000030 & 13 \\
\hline & 38 & 15,801281 & 0,000042 & 7 & 15,801173 & 0,000037 & 12 \\
\hline No & gyen & 7,900716 & 0,000015 & 126 & 7,900598 & 0,000014 & 137 \\
\hline
\end{tabular}

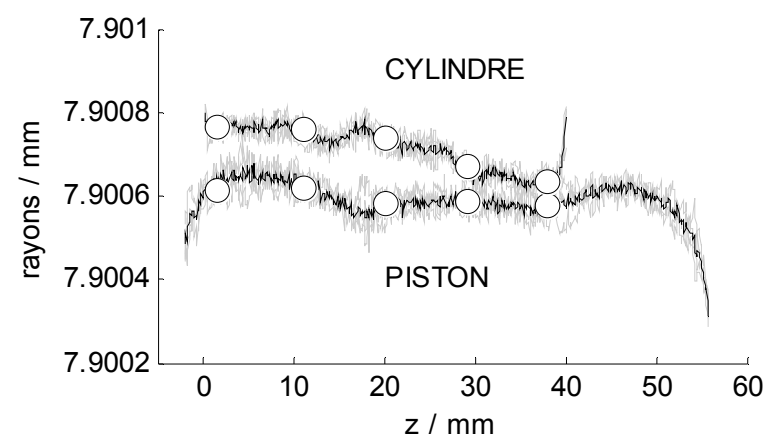

Figure 3. Profil du jeu de l'ensemble piston-cylindre $50 \mathrm{MPa}$ $\mathrm{N}^{\circ} 1(-)$ et positionnement des demi-diamètres ( $(0)$.

\section{Comparaison des sections}

Le piston-cylindre $50 \mathrm{MPa} \mathrm{N}^{\circ} 1$ a été étalonné par comparaison directe avec l'ensemble piston-cylindre Desgranges et Huot $\mathrm{N}^{\circ}$ 6594, dont la valeur de section est référencée dans la base de données du BIPM et est issue de l'intercomparaison CCM.P-K1 [3][4], sa valeur est égale à 980,492 $9 \mathrm{~mm}^{2}$. Cette comparaison a été effectuée en gaz pur pour des pressions comprises entre 0,1 et $1 \mathrm{MPa}$. Cette mesure nous a permis de trouver une section effective à pression nulle égale 196,098 $90 \mathrm{~mm}^{2}$ avec une incertitude type de $5,5 \times 10^{-6} \times S$. Soit un écart relatif de $3,6 \mathrm{ppm}$ avec la valeur déterminée précédemment par mesures dimensionnelles.

Cette nouvelle référence $50 \mathrm{MPa}$ se compose de trois ensembles pistons-cylindre que nous avons comparés deux à deux et par rapport au $200 \mathrm{MPa} \mathrm{N}^{\circ} 5$. Les résultats montrent des écarts relatifs sur la section inférieurs à 3,1 ppm (Tableau 4).
Tableau 4. Comparaison des sections $\left(\mathrm{mm}^{2}\right)$ des ensembles piston-cylindre $50 \mathrm{MPa}$ et raccordement à l'ensemble $200 \mathrm{MPa}$ $\mathrm{N}^{\circ} 5$.

\begin{tabular}{cccc}
\hline $\begin{array}{c}50 \mathrm{MPa} \\
\mathrm{N}^{\circ}\end{array}$ & $\begin{array}{c}50 \mathrm{MPa} \\
\mathrm{N}^{\circ} 1\end{array}$ & $\begin{array}{c}200 \mathrm{MPa} \\
\mathrm{N}^{\circ} 5\end{array}$ & $\begin{array}{c}\text { Ecart } \\
\text { ppm }\end{array}$ \\
\hline 2 & 196,10558 & 196,10498 & 3,1 \\
3 & 196,10460 & 196,10407 & 2,7 \\
\hline \hline
\end{tabular}

Pour compléter la boucle d'étalonnage, nous avons comparé le piston $50 \mathrm{MPa} \mathrm{N}^{\circ} 2$ avec le $\mathrm{N}^{\circ} 3$ et nous trouvons une section sur l'ensemble $\mathrm{N}^{\circ} 2$ de $196,10474 \mathrm{~mm}^{2}$, soit un écart relatif de 6,2 ppm.

Nous avons effectué le raccordement de nos ensembles de référence haute pression $200 \mathrm{MPa}$ à partir le l'ensemble piston cylindre $50 \mathrm{MPa} \mathrm{N}^{\circ} 1$ pour lequel nous avons choisi comme valeur de référence la section effective déterminée par mesures dimensionnelles. Nous avons trouvé des valeurs qui sont cohérentes avec les raccordements effectués en 1978 (Tableau 5) avec des écarts relatif inférieurs à $5 \mathrm{ppm}$. Seul l'ensemble $200 \mathrm{MPa} \mathrm{N}^{\circ} 2$ donne des écarts pouvant atteindre $11,1 \mathrm{ppm}$.

L'incertitude-type sur la détermination de la section des ensembles piston-cylindre $200 \mathrm{MPa}$ à partir de l'ensemble $50 \mathrm{MPa} \mathrm{N}^{\circ} 1$ est de 7,0 x $10^{-6} \times S$.

\section{Coefficient de déformation}

Autant la section effective à pression nulle doit être déterminée à basse pression avec des ensembles pistoncylindre de grand diamètre, autant il est préférable de déterminer les coefficients de déformation à partir des 
pressions beaucoup plus élevées. C'est pourquoi, nous avons déterminé le coefficient de déformation de l'ensemble $50 \mathrm{MPa}$ à partir de l'ensemble $200 \mathrm{MPa} \mathrm{N}{ }^{\circ} 5$ dont la valeur du coefficient de déformation est issue des travaux menés par Legras en 1978 [5] et confirmée par de nombreuses comparaisons internationales dont la plus récente est la CCM.P-K7 (2005) [6]. Nous obtenons ainsi un coefficient de déformation égal à $1,13 \times 10^{-6} \mathrm{MPa}^{-1}$.

\section{7 Étude théorique}

Pour calculer le coefficient de déformation, nous pouvons utiliser les équations de l'élasticité [7] en supposant que l'ensemble piston-cylindre est parfaitement cylindrique et que le champ de pression est linéaire ; nous obtenons :

$$
\lambda=\frac{\bar{u}_{p}+\bar{U}_{p}}{r_{0}} \frac{1}{P},
$$

avec $\bar{u}_{p}$ et $\bar{U}_{p}$ les déformations moyennes sous pression :

$$
\begin{gathered}
\bar{u}_{p}=\frac{r_{0} P}{2 E}(3 \mu-1), \\
\bar{U}_{p}=\frac{R_{0} P}{2 E}\left[\frac{R_{j}^{2}+R_{0}^{2}}{R_{j}^{2}-R_{0}^{2}}+\mu\right] .
\end{gathered}
$$

E est le module d'Young, $\mu$ le coefficient de Poisson, R0 le rayon interne du cylindre qui est égal au rayon à l'entrée du cylindre $\mathrm{r} 0$ auquel on ajoute le jeu à l'entrée $\mathrm{h} 0$ et $\mathrm{Rj}$ le rayon externe du cylindre.

Les ensembles piston-cylindre ont été réalisés en carbure de tungstène avec $6 \%$ de cobalt. Les constantes $E$ et $\mu$ ont été préalablement mesurées sur les épures des pistons et cylindres à l'aide de jauges de déformation. Les valeurs expérimentales sont présentées dans le tableau 6 .

Ces valeurs conduisent, d'après l'équation (16), à un coefficient de déformation théorique $\lambda_{\text {th. }}=1,19 \times 10^{-6} \mathrm{MPa}^{-1}$.
Tableau 6. Module d'Young et coefficient de Poisson des pistons et cylindres $50 \mathrm{MPa} \mathrm{N} \mathrm{N}^{\circ}$.

\begin{tabular}{ll}
\hline \hline Piston & $\mathrm{E}=(628,3 \pm 8,0) \mathrm{GPa}$ \\
& $\mu=(0,213 \pm 0,003)$ \\
Cylindre & $\mathrm{E}=(626,4 \pm 3,8) \mathrm{GPa}$ \\
& $\mu=(0,211 \pm 0,002)$ \\
\hline \hline
\end{tabular}

Une autre méthode consiste à calculer les déformations par la méthode par éléments finis [8][9]. Cette méthode permet d'intégrer des géométries de structures plus complexes et de mieux maîtriser les conditions aux limites, comme la position des joints d'étanchéité ou le champ de pression. Les calculs ont été réalisés sous Matlab avec le module Structural Dynamics Toolbox (SdTools).

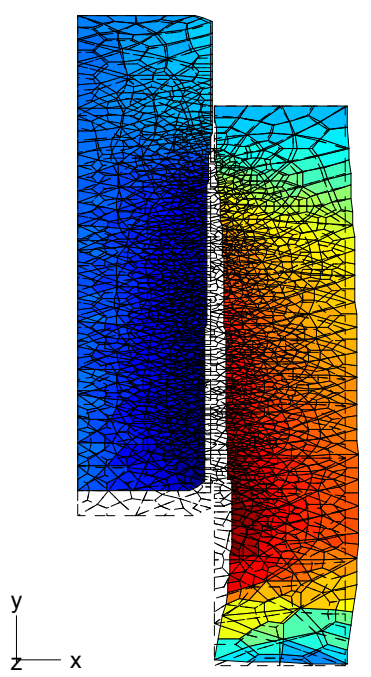

Figure 4. Maillage et déformations radiales calculés avec SdTools sur l'ensemble piston-cylindre $50 \mathrm{MPa} \mathrm{N}^{\circ} 1$.

\begin{tabular}{|c|c|c|c|c|c|}
\hline \multirow{2}{*}{$\begin{array}{c}\text { Ensemble } \\
\text { Piston-cylindre }\end{array}$} & \multirow{2}{*}{$\begin{array}{c}\text { Dimensionnel } \\
1978\end{array}$} & \multirow{2}{*}{$\begin{array}{c}\text { Dimensionnel } \\
2009\end{array}$} & \multirow{2}{*}{$\begin{array}{c}\text { Comparaison } \\
\text { section } \\
1978\end{array}$} & \multicolumn{2}{|c|}{ Comparaison section 2012} \\
\hline & & & & $50 \mathrm{MPa} \mathrm{N}^{\circ} 1$ & $200 \mathrm{MPa} \mathrm{N}^{\circ} 5$ \\
\hline $200 \mathrm{MPa} \mathrm{N}^{\circ} 2$ & $\overline{50,2744}$ & & 50,2735 & $\begin{array}{c}50,27392 \\
(8,3)\end{array}$ & $\begin{array}{c}50,27406 \\
(11,1)\end{array}$ \\
\hline $200 \mathrm{MPa} \mathrm{N} 3$ & 50,2740 & & 50,2742 & $\begin{array}{c}50,27435 \\
(2,9)\end{array}$ & $\begin{array}{c}50,27435 \\
(2,9)\end{array}$ \\
\hline $200 \mathrm{MPa} \mathrm{N}^{\circ} 4$ & 50,2773 & $\begin{array}{c}50,27726 \\
(0,8)\end{array}$ & 50,2765 & $\begin{array}{c}50,27626 \\
(-4,9)\end{array}$ & $\begin{array}{c}50,27638 \\
(-2,3)\end{array}$ \\
\hline $200 \mathrm{MPa} \mathrm{N}^{\circ} 5$ & 50,2729 & & 50,2719 & $\begin{array}{c}50,27182 \\
(-1,6)\end{array}$ & \\
\hline $200 \mathrm{MPa} \mathrm{N}^{\circ} 6$ & 50,2737 & & 50,2733 & $\begin{array}{c}50,27335 \\
\quad(1,0)\end{array}$ & $\begin{array}{c}50,27336 \\
(1,2)\end{array}$ \\
\hline
\end{tabular}

Tableau 5. Comparaison des sections effectives $\left(\mathrm{mm}^{2}\right)$ des ensembles piston-cylindre mesurées à partir de l'ensemble $50 \mathrm{MPa} \mathrm{N}^{\circ} 1$ et des mesures dimensionnelles, avec entre parenthèse les écarts relatifs par rapport aux résultats de 1978 en ppm. 
Tableau 7. Bilan d'incertitude sur la détermination de la pression pour les ensembles piston-cylindre 50 MPa $\mathrm{N}^{\circ} 1$.

\begin{tabular}{|c|c|c|c|}
\hline Composante & $\begin{array}{c}\text { Incertitude- } \\
\text { type sur la } \\
\text { composante } \\
\end{array}$ & Unité & Incertitude-type \\
\hline $\begin{array}{l}\text { Répétabilité } \\
\text { Masse } \\
\text { Stabilité des masses } \\
\text { Mo (force des fils d'alimentation du moteur entrainement } \\
\text { du piston) } \\
\text { Masse volumique des masses } \\
\text { Masse volumique du piston } \\
\text { Section effective à pression nulle et à }+20^{\circ} \mathrm{C} \\
\text { Coefficient de déformation } \\
\text { Température } \\
\text { Coefficients de dilatation }\left(20 \pm 1,5^{\circ} \mathrm{C}\right) \\
\text { Verticalité } \\
\text { Masse volumique de l'air } \\
\text { Masse volumique de l'huile } \\
\text { Définition hauteur de référence (mesure) } \\
\text { Hauteur de référence (mesure) } \\
\text { Position du piston } \\
\text { Accélération de la pesanteur }\end{array}$ & $\begin{array}{c}4,1 \times 10^{-4} \\
5,1 \times 10^{-4} \\
5,0 \times 10^{-5} \\
1,98 \\
65 \\
5,5 \times 10^{-6} \\
5,7 \times 10^{-14} \\
5,8 \times 10^{-2} \\
1,2 \times 10^{-7} \\
3,4 \times 10^{-4} \\
4,0 \times 10^{-4} \\
5 \\
0,50 \\
0,33 \\
0,03 \\
9,8 \times 10^{-7}\end{array}$ & $\begin{array}{c}\mathrm{kg} \\
\mathrm{kg} \\
\mathrm{kg} \\
\mathrm{kg} / \mathrm{m}^{3} \\
\mathrm{~kg} / \mathrm{m}^{3} \\
\mathrm{X} \mathrm{S}^{3} \\
\mathrm{~Pa}^{-1} \\
{ }^{\circ} \mathrm{C} \\
{ }^{\circ} \mathrm{C}^{-1} \\
\mathrm{rad} \\
\mathrm{kg} / \mathrm{m}^{3} \\
\mathrm{~kg} / \mathrm{m}^{3} \\
\mathrm{~mm} \\
\mathrm{~mm} \\
\mathrm{~mm} \\
\mathrm{~m} / \mathrm{s}^{2}\end{array}$ & $\begin{array}{c}3,3 \mathrm{~Pa}+1,0 \times 10^{-6} \times \mathrm{p} \\
4,0 \times 10^{-7} \times \mathrm{p} \\
5,0 \times 10^{-7} \times \mathrm{p} \\
3 \mathrm{~Pa} \\
3,8 \times 10^{-8} \times \mathrm{p} \\
5,5 \times 10^{-6} \times \mathrm{p} \\
5,7 \times 10^{-14} \times \mathrm{p}^{2} \\
5,2 \times 10^{-7} \times \mathrm{p} \\
1,8 \times 10^{-7} \times \mathrm{p} \\
1,1 \times 10^{-7} \times \mathrm{p} \\
5,1 \times 10^{-8} \times \mathrm{p} \\
2,5 \mathrm{~Pa} \\
4 \mathrm{~Pa} \\
3 \mathrm{~Pa} \\
0,30 \mathrm{~Pa} \\
1,0 \times 10^{-7} \times \mathrm{p}\end{array}$ \\
\hline
\end{tabular}

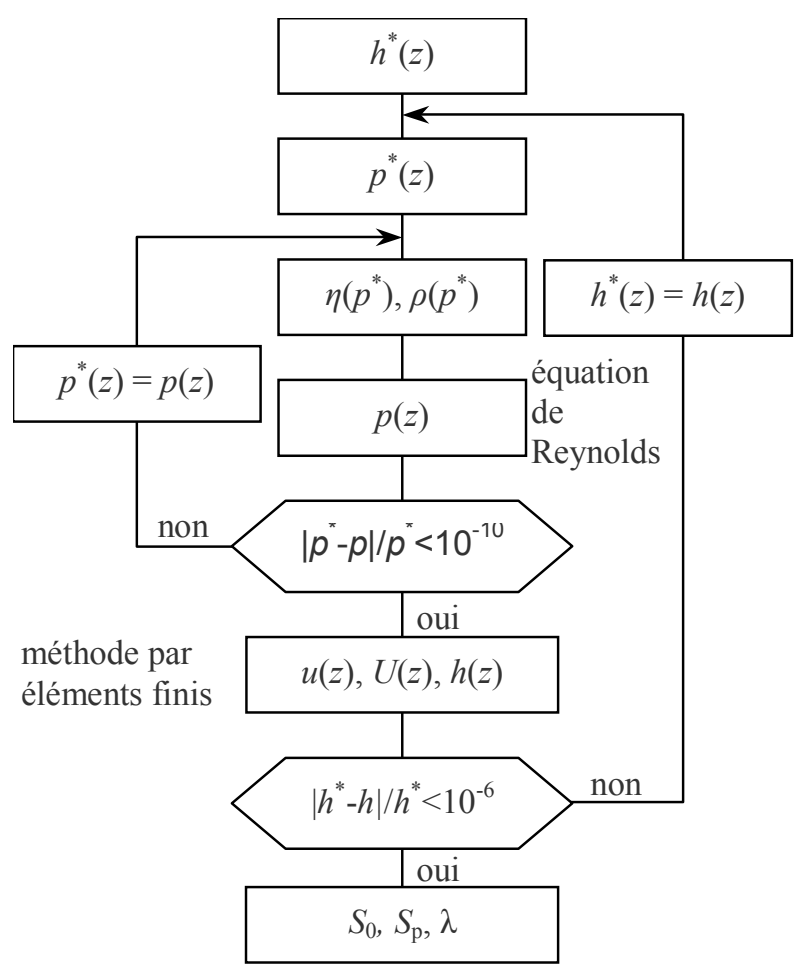

Figure 5.Méthode de calcul des coefficients de déformation par la méthode des éléments finis.

Le calcul consiste à partir du jeu initial $h^{*}$ et après avoir initialisé le champ de pression $p^{*}$ à calculer la viscosité $\eta\left(p^{*}\right)$ pour en déduire le champ de pression $p^{*}$ à partir de l'équation de Reynolds (13). Ce nouveau champ de pression servira à recalculer la viscosité jusqu'à convergence du champ de pression. Ce champ de pression sera appliqué aux parois du piston et cylindre dans le jeu, afin de calculer leurs déformations respectives $u$ et $U$ par la méthode des éléments finis. Nous utiliserons ces déformations pour calculer le nouveau jeu $h$ que nous utiliserons pour le calcul du champ de pression jusqu'à convergence du jeu. À partir des déformations ainsi obtenues, nous pouvons en déduire les sections effectives $S_{0}$ et $S_{\mathrm{p}}$ et le coefficient de déformation $\lambda$, à l'aide des équations (14), (12) et (2). Cette méthode est schématisée dans la figure 5 .

Le fluide utilisé dans nos balance est du di(2 ethylhexyl) sebacate dont les caractéristiques ont été mesurées par Vergne [10] et sont données par les équations (19) et (20), où $\mathrm{p}$ est exprimé en $\mathrm{MPa}, \eta$ en Pa.s et $\rho$ en $\mathrm{kg} / \mathrm{m}^{3}$.

$$
\begin{array}{r}
\eta(p)=0,021554\left(1+1,90036 \cdot 10^{-3} p\right)^{8,8101} \\
\rho(p)=912,6657+0,752097 p-1,64485 \cdot 10^{-3} p^{2} \\
\cdots+1,45625 \cdot 10^{-6} p^{3}
\end{array}
$$

Les calculs ont été effectués en prenant en compte le jeu réel du piston-cylindre $50 \mathrm{MPa} \mathrm{N}^{\circ} 1$ (Figure 3) dans lequel nous observons une conicité côté cylindre de $-2,82 \times 10^{-6}$.

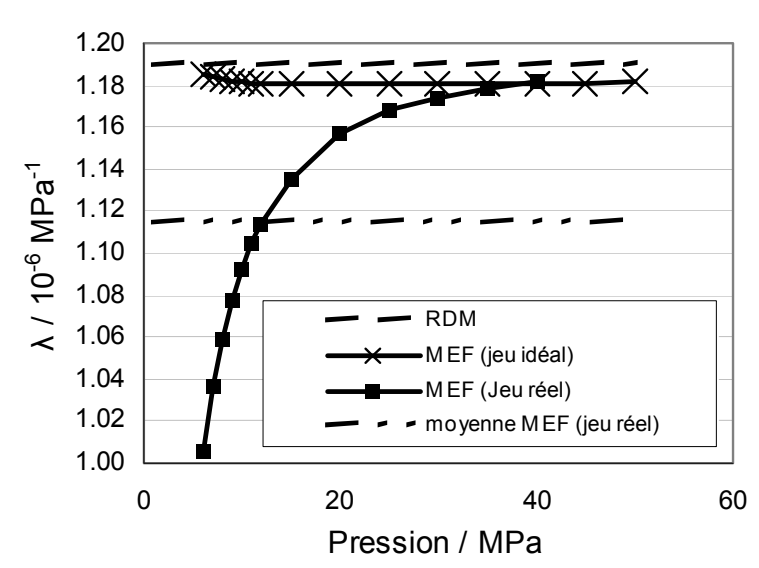

Figure 6.Évolution du coefficient de déformation de $l^{\prime}$ ensemble piston-cylindre $50 \mathrm{MPa} \mathrm{N}^{\circ} 1$. Incertitudes 
Cette conicité a pour effet de rendre le coefficient de déformation non linéaire (Figure 6). Sa valeur varie entre 1 et $1,18 \times 10^{-6} \mathrm{MPa}^{-1}$. Si nous faisons la moyenne des coefficients de déformation calculée au niveau des paliers de mesure, nous obtenons une valeur de 1 égale à $1,145 \times 10^{-6} \mathrm{MPa}^{-1}$ proche de $1,13 \times 10^{-6} \mathrm{MPa}^{-1}$ trouvée expérimentalement, soit un écart relatif de $1,3 \%$.

\section{Incertitudes}

Pour effectuer des comparaisons à partir des ensembles piston-cylindre $50 \mathrm{MPa}$, nous devons établir un bilan d'incertitude sur la détermination de la pression.(Tableau 7).

Nous constatons que pour une pression de $50 \mathrm{MPa}$, l'incertitude sur la section effective représente $64 \%$ de l'incertitude-type composée, qui est de $7 \mathrm{~Pa}+5,7 \times 10^{-}$ ${ }^{6} \mathrm{xp}+1,1 \times 10^{-13} \mathrm{xp}^{2}$. L'incertitude sur la pression due au coefficient de déformation représente $32 \%$ de l'incertitude globale et $12 \%$ pour la répétabilité.

Nous pouvons ainsi déclarer pour nos CMC (Calibration and Measurement Capability) une incertitude élargie $(k=2)$ de $15 \mathrm{~Pa}+1,2 \times 10^{-5} \times \mathrm{p}+$ $1,1 \times 10^{-13} \times \mathrm{p}^{2}$.

\section{Conclusion}

Les ensembles piston-cylindre $50 \mathrm{MPa}$ ont démontré qu'ils fonctionnent aussi bien en gaz qu'en huile.

Leurs raccordements directs aux unités SI en font des étalons primaires de pression qui viennent enrichir la gamme de notre référence haute pression. Les mesures dimensionnelles nous ont données une incertitude sur la section effective à pression nulle inférieure à $10 \mathrm{ppm}$ $(2 \sigma)$. Ces valeurs ont été confirmées par le raccordement en basse pression avec nos étalons $10 \mathrm{~cm}^{2}$ et ont montré une continuité avec nos références $200 \mathrm{MPa}$.

La détermination du coefficient de déformation effectuée sur l'ensemble $50 \mathrm{MPa} \mathrm{N}^{\circ} 1$ a montré une cohérence entre mesure et expérimentation.

La nouvelle chaîne de référence $50 \mathrm{MPa}$ est maintenant opérationelle avec une incertitude de $15 \mathrm{~Pa}+1,2 \times 10^{-}$ ${ }^{6} \mathrm{xp}+1,1 \times 10^{-13} \mathrm{x} \mathrm{p}^{2}$ valeur déclarée pour nos capacités de mesure et d'étalonnage (CMC).

\section{Références}

1. J.C. Legras, A. Huot, P. Delajoud, "La référence nationale de pression du BNM dans le domaine de 5 à $200 \mathrm{MPa}$ ", Bull. d'Information du BNM, (avril 1982), 48, pp. 9-33.

2. S.L. Dadson, S.L. Lewis, G.N. Peggs, "The Pressure Balance - Theory and Practice", Her Majesty's Stationnary Office, NPL, (1982)

3. G.F. Molinar et al., "CCM key comparison in the pressure range $0.05 \mathrm{MPa}$ to $1 \mathrm{MPa}$ (gas medium, gauge mode). Phase A1: Dimensional measurements and calculation of effective area", Metrologia, (1999), 36, p. 657

4. J. C. Legras et al., "CCM key comparison in the pressure range $50 \mathrm{kPa}$ to $1000 \mathrm{kPa}$ (gas medium, gauge mode). Phase A2: Pressure measurements",

Metrologia, (1999), 36, 663

5. J.C. Legras, "Etude métrologique d'une référence de pression dans le domaine de 5 à $200 \mathrm{MPa}$ ", Mémoire d'ingénieur CNAM, (1978)

6. W Sabuga, M Bergoglio, T Rabault, B Waller, J C Torres, D A Olson, A Agarwal, T Kobata and A K Bandyopadhyay, "Final Report on Key Comparison CCM.P-K7 in the range $10 \mathrm{MPa}$ to $100 \mathrm{MPa}$ of hydraulic gauge pressure",Metrologia, 42, (2005),

7. S.P. Timoshenko, "Résistance des matériaux", Dunod, Tome 2, (1930), 213-220

8. T. Rabault et J.C. Legras, "Calculation of the pressure distortion coefficient and uncertainty budget of the $B N M / L N E 200 \quad M P a$ national standard", Metrologia, 42, (2005), pp. 246-249

9. W. Sabuga, G. Molinar, G. Buonanno, T. Esward, T. Rabault et L. Yagmur, "Calculation of the distortion coefficient and associated uncertainty of PTB and LNE $1 \mathrm{GPa}$ pressure balances using finite element analysisEUROMET project 463", Metrologia, 42, (2005), pp. 202-206.

10. P. Vergne, "New high-pressure viscosity measurements on di(2-ethylhexyl) sebacate and comparisons with previous data", (1990), High Temperatures - High Pressures, 22, nº, pp. 613-621 